\title{
Decision Support for Healthcare ICT Network System Appraisal
}

\author{
A.M. Oddershede, L.E. Quezada, F.M. Cordova, R.A. Carrasco
}

\author{
Astrid M. Oddershede, Luis E. Quezada, \\ Felisa M. Cordova \\ University of Santiago of Chile, \\ Industrial Engineering Department \\ Av. Ecuador 3769, Santiago, Chile \\ E-mail: astrid.oddershede@usach.cl, \\ luis.quezada@usach.cl,felisa.cordova@usach.cl
}

Rolando A. Carrasco

Newcastle University, UK, School of IEEE

Newcastle Upon Tyne, UK

E-mail: r.carrasco@ncl.ac.uk

\begin{abstract}
:
A framework to support the appraisal process to improve the quality of service (QoS) of an Information and Communication Technology (ICT) network system in health care service is presented. Most of health-related activities stand to benefit from ICT endorsement; however, technical problems may appear, as an inadequate physical infrastructure, insufficient access by the user to the hardware/software communication infrastructure and QoS issues. The aim is to develop a prototype assessment model based on data collected from the main users of a health network system An evaluation process is carried out to analyze and assess the support of QoS of ICT, its infrastructure and user interface perception of the QoS offered through case study for hospitals in Chile. Performance has been evaluated by simulation and modelling network Architecture. The Optimization Network Engineering Tool (OPNET) simulation platform is used to examine the network behaviour and performance to ensure consistency and reliability for thousands of staff across the hospital network.
\end{abstract}

Keywords: ICT, Healthcare, OPNET, MCDM.

\section{Introduction}

Nowadays, Healthcare Institutions are universally urged to improve quality of their services and Hospital units are concentrated on how to develop better services, to allocate methods, to provide resources to satisfy professional aspirations and to comply with citizen necessities. Most of the actions oriented to improve the operation and the quality of healthcare service depends, to a great extent, on the level of information available and the communications system. A poor ICT network system implementation may generate a negative effect on the service, patients and health care providers.

Furthermore, the ICT system from a health centre may be endowed by attributes that not often matches with the user's requirement s (quality level, performance, cost and others). The ICT system may be inappropriate for satisfying user requirements or may be inefficient in doing it. ICT system should be a facilitator for health care users since they need to access to all types of data existing on all types of systems.

The challenge of providing quality of service (QoS) in a health environment is further complicated by the extremely variable QoS needs of individual health organizations over time. The kinds of information exchanges in which an organization engages typically vary considerably in the course of a day, from simple exchanges of information regarding a patient's coverage by a health plan, through transfers of medical records with affiliated organizations, to the exchange 
of large medical images for interpretation and diagnosis. For example, the bandwidth needs of a small medical clinic could, accordingly, vary enormously during the course of a day, ranging from near nothing one minute to several megabits per second the next. Finding ways to satisfy such variable demand for bandwidth economically represents a significant challenge.

The agents (patient, clinical doctors, hospital staff, government, etc) involved should attend contradictory petitions: on one hand, to respond the increasing demand and, by another, to attend to the budgetary restrictions.

The answer to this complexity is to improve QoS and the efficiency [1] [2] . User needs and expectations are indispensable to count on better information that lead to an improvement in operation services, welfare and clinical information. Reorienting the Health care system towards the needs of the client, medicine based on the evidence, clinical management and the continuous improvement of the processes (QoS), Total quality, contemplating the human factor as the main assets of the business) are key elements in future.

The hospital management challenge is based on the achievement of the articulation and the convergence of values among the agents, the staff members and the patients; the Information Technology and Communications Systems needed are not just mere data agents, instead, they are of information, knowledge and intellectual capital [3] Some general questions to be answered are: What are user expectations about the ICT needs? Is the current ICT infrastructure appropriate? What are the main QoS parameters? How can be evaluated the ICT network for Healthcare service? What technical capabilities do health applications demand of the Internet? How do these capabilities differ from those needed by applications in other sectors, such as banking, defense, and entertainment?.

To deal with this complexity, a two stages methodology involving user perception is proposed. The first stage corresponds to the identification of the user types, the activities they are involved in, the ICT network system requirements, applications and priorities attributes. This is achieved through Multicriteria Decision Making approach (MCDM) [4], using the Analytical Hierarchy Process (AHP) in author previous work [8] obtaining key QoS parameters.

This paper focuses on the second stage, which is the development of a model for evaluating ICT healthcare network quality of service (QoS) integrating user perception. This stage is concerned with modelling and simulation to examine the network performance on applications. The QoS technical metrics related to each attribute has to be defined together with the applications profile. QoS offered by a particular network could be established by technical parameters that can be measured objectively. However, user perception depends upon their needs, their precise applications and their expectations. It is a difficult task to find a set of universal parameters for every type of service because there are many and dissimilar parameters involved in the performance evaluation.

A typical public hospital zone is considered as a case study to analyze technology infrastructure and network performance. Further, profile applications must be set up according to the main role health care users perform. A new model based on the results obtained on the first stage is designed and OPNET simulation platform is used to examine the network behaviour and performance .The model incorporates communications resources of LAN architecture from typical Chilean hospitals where. OPNET model has demonstrated to give a good representation to real world to analyze traffic flows, and network performance providing a tool to demonstrate different type of networks and protocols.

This pilot case study revealed that the model shows to be useful by evaluating quality, user pertinent criteria and to connect higher objectives with lower performance metrics and conclude that through the proposed assessment model is possible to detect whether there is connection between human perception of QoS and the technical metrics associated to the ICT network. This analysis helps decision makers, network planners and operations engineers to 
manage complex and constantly changing networks, using predictive planning for reacting to significant network issues using real-time network visualization and troubleshooting. This is critical in a healthcare institution.

Section 2 provides a description of the conceptual model, in section 3 the case study description is presented .The simulation results generate new information, and section 5 provides the conclusions.

\section{Conceptual Model for Healthcare Service ICT Network}

A conceptual model is proposed for health ICT network system evaluation connecting QoS user perception (qualitative) with QoS technical aspects, (parameters, and network performance). Modeling and simulation provides a good tool, to plan network infrastructure and manage application performance using predictive planning. The model scheme is depicted through Figure 1 illustrating the diverse factors involved in the evaluation of QoS for a particular service starting from the end user. Initially, the main users, the type of user and its applications must be categorized, then the ICT support needs to deliver a better service, the attributes indispensable to meet the requirement in concordance to their expectation, are to be characterized, next , technical metrics should be defined and analyzed to check the performance on an application [5].

The perceived QoS cluster includes the parameters related to each service the user perceive and determine the satisfaction of the service received. For example: success in the connection, accessibility, velocity, etc. The technical ICT parameters refers to the basic metrics that would guarantee a service, i.e., end to end delay, packet loss and should be detected by the network operators in charge.

QoS offered by a particular network could be established by technical parameters that could be measured objectively. However the user perception depends upon their needs, their directly application and their expectations. It is a difficult task to find a set of universal parameters for every type of service for the reason that there are many and dissimilar parameters involved in the performance evaluation. Then, it is useful to analyze which of the parameters is relevant when considering the user perception for a determined service. The organization must then define a service level agreement (SLA) for their main applications.

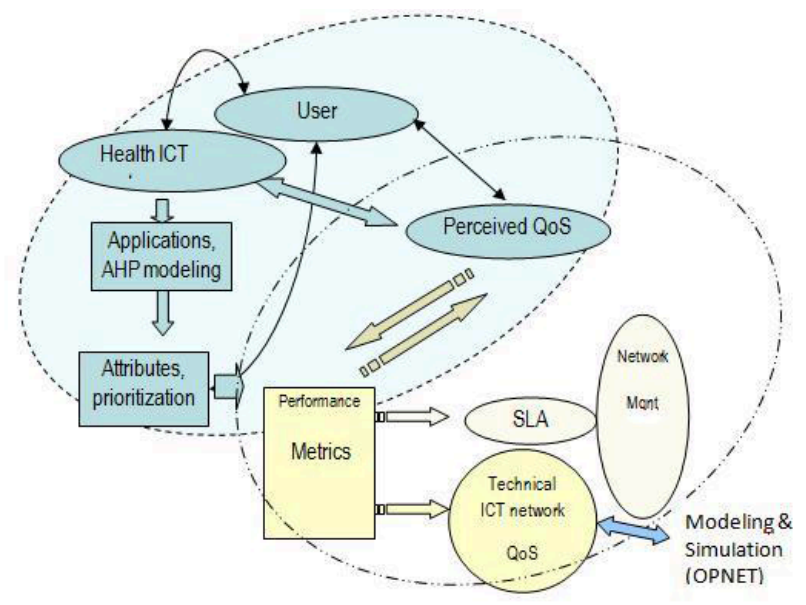

Figure 1: Proposed Conceptual model for health ICT network evaluation 
For the case in study, we suggest modeling and simulation using Optimization Network Engineering Tool (OPNET) simulation platform, (www.opnet.com). [6], [7] to examine the network behavior and performance when it is not possible to compute through the operators the basic metrics. OPNET supports simulation technologies and it is well suited to examining network behavior.

The OPNET models execute the protocol in much the same way as a production environment. The modeling it is based on Cisco network which is $99 \%$ accurate and good representation of real world. [8] It is possible to study protocol behavior under different network conditions and application performance.

\section{Hospital ICT Network Scenarios Simulations with OPNET}

A pilot case study is pursued following the methodology proposed, where the results can be used for analyzing the network performance en ICT healthcare system, when it is not possible to measure directly, the performance metrics, and parameters.

According to OPNET methodology, the initial phase for analyzing the effect of QoS on an ICT network and/or on application performance is to select a network topology, followed by traffic configuration for each application before running simulations. Then QoS has to be configured defining SLAs to analyze the results.

\subsection{Hospital Zone Case Study}

The authors have collected information from a Public Community hospital/medical centre/clinic Hospital Dr. Luis Tisne Brousse in Santiago Chile. A Local Ethernet network from a zone of the medical centre is considered as an initial pilot study case. In view of the fact that the purpose is to examine the backbone utilization, the study zone is represented as a partial network topology along with the methodology.

\section{Hospital Zone Description}

The backbone Giga Bit Ethernet in study is located at a building next to the hospital where they perform administrative functions to assist the hospital and some health service functions.

A diagram showing the zone in study is given in Figure 2.

The section in study is a two floor building, plus a basement named: "Zócalo". Within the first floor, there are rooms used for providing diagnosis for minor ailments and medical consultation. This first floor includes 3 switches:

- WS2950G-24, switch, located at the Electric room, with five workstations,

- WS2950-24, switch located at the medicine/Physiatrist room, with six work stations,

- WS2950G-12 located at the X ray room, with 3 workstations.

- The basement floor includes a WS2950G-24, Switch with 24 ports and there are 6 workstations connected,

- The first floor switches are connected to the second floor to a WS2950G-24 switch located at the Director office by fibber optical cable. The switches are connected to and between each other by Optic fiber, Except for the X ray room switch which is a cable UTP cat. 5E. 


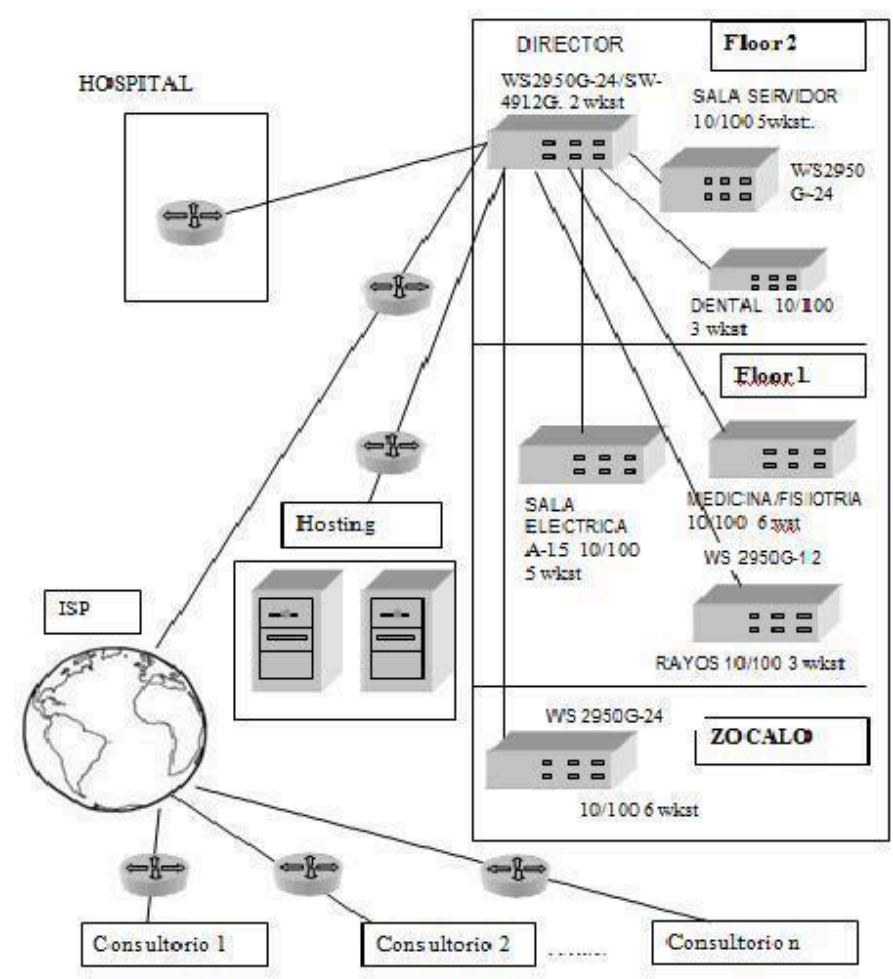

Figure 2: Hospital zone diagram

\subsection{OPNET Network Modeling for Public Hospital Zone Case Study}

The authors have designed network modeling and implementation for the public Hospital stated in conformity with the diagram described on figure 4. The partial network topology is represented following OPNET methodology in Figure 3.

The partial network characterizes the initial situation and first scenario to be explored. A total of 30 workstations are initially linked to the switches. The different services the users deliver are expected to be supported by the network through different applications that will help to accomplish their assignment. The applications regard as sustained by the server are based on the results obtained on the previous study.

\subsection{Health ICT Network Applications}

Communications networks enable applications to exchange data. Popular applications that use data networks include virtual terminal services, file transfer utilities, database transactions, and e-mail. Each of these applications generates its own sort of traffic: virtual terminals slowly generate many small packets, while file transfer utilities send long streams of large packets.

Each type of traffic causes and experiences a different set of problems in the underlying network, so you may want to accurately model the traffic patterns generated by a variety of applications. OPNET uses a generic network application model to generate typical application traffic patterns. This is the applications model, also called the standard network application model.. Depending on their underlying networks, application architectures may differ [7].

The applications are modeled explicitly, and end-to-end delays or response times are studied in detail. The factors that contribute to application response time include,: 


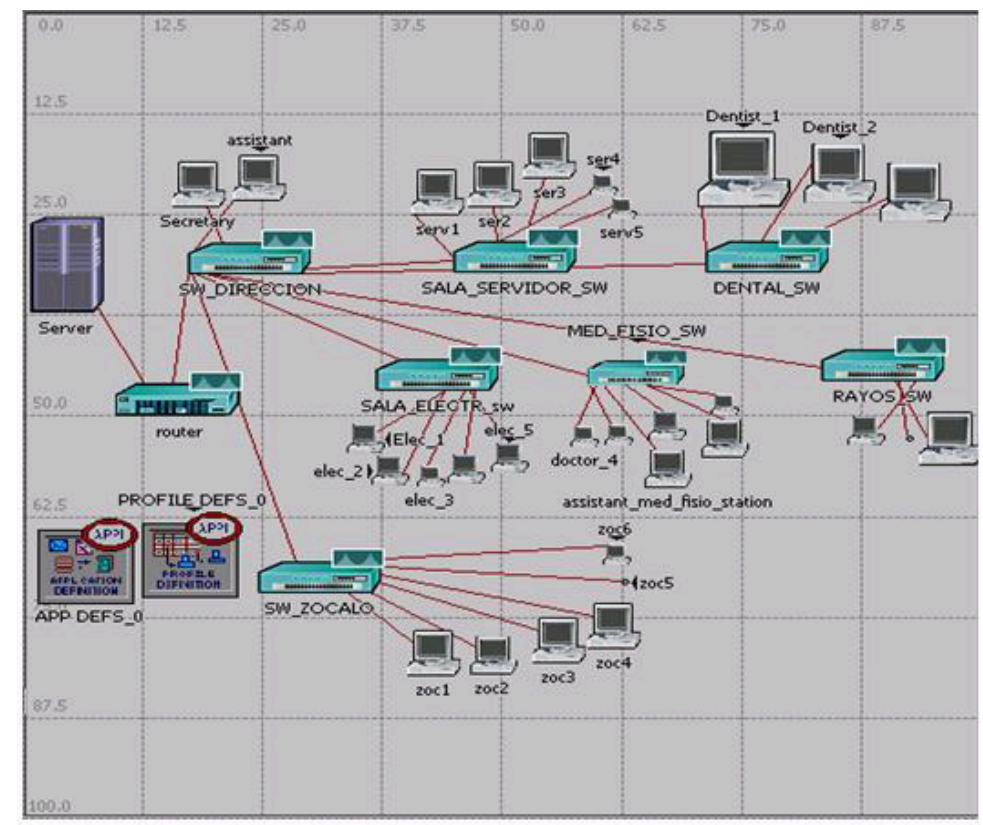

Figure 3: Partial network topology

- Delays due to contention on servers (server processing time),or/and

- Delays due to contention on the client (client processing time),

- Delays due to contention with "other" traffic at the various intermediate devices (queuing delays),

- Delays due to contention with traffic of the same application type from other users at the intermediate devices (queuing delays),

- Network delays (transmission and propagation),

- Delays due to protocol effects (TCP retransmissions, windowing etc.)

In the course of the investigation and survey developed on a previous work [9], [10], the findings revealed that the relative usage of ICT applications in health care differs to some extent depending on the institution. Indicating that, so far, the real application that has more usage is e- mail and there is very little usage of the others. Web browsing is used mainly on research activities.

However, through the multicriterial analysis by the use of AHP, to find out the importance of ICT provision in quality of service in healthcare institutions developed in[10], one of the important new information, conclusions and contribution are the relative importance users assigned to the applications in performing a health care service. User assigned the highest priority in importance to data base access and the least relative importance to e-mail. This shows the existence of a gap between what healthcare participants actively use and what they consider important for the development of their daily work as a service for the patients.

Then, considering these results the applications to be supported by the server for every scenario are: Email, Web Browsing (Http 1.1), File transfer, Database Access, File Print, Video Conferencing, and Voice. 


\subsection{Applications Configuration}

A profile is applied to each workstation, server, or LAN. It specifies the applications used by a particular group of users. An application may be any of the common applications, email, file transfer, etc., that may be defined. The next step is to set up profile applications according to each user type. Every workstation will have a profile application consistent with the users' main role.

After the participants have studied the nature of the system, then profiles are designed with respect to the functions a user makes use of in/at a health service. This profile name is assigned for classification. These applications profiles were defined using the results from the analytic hierarchy process.

Once applications and profiles are defined, it is possible to characterize different scenarios for each study case intended for visualizing how sensible is a networks performance with respect to changes.

\section{$4 \quad$ Simulation Results}

Different scenarios for the study case are characterized to determine the sensitivity of main key parameters (as throughput and utilization). Initially, the applications described are modeled explicitly and all background traffic is disabled. Simulations are ran for each scenario, increasing the number of users and their respective applications. Through Figure 4 it is possible to visualize overlaid run results for delay average (in Ethernet. delay (secs) for four scenarios. As the number of users is increased, the average delay time increases at different rates, until it tends to be stabilized.

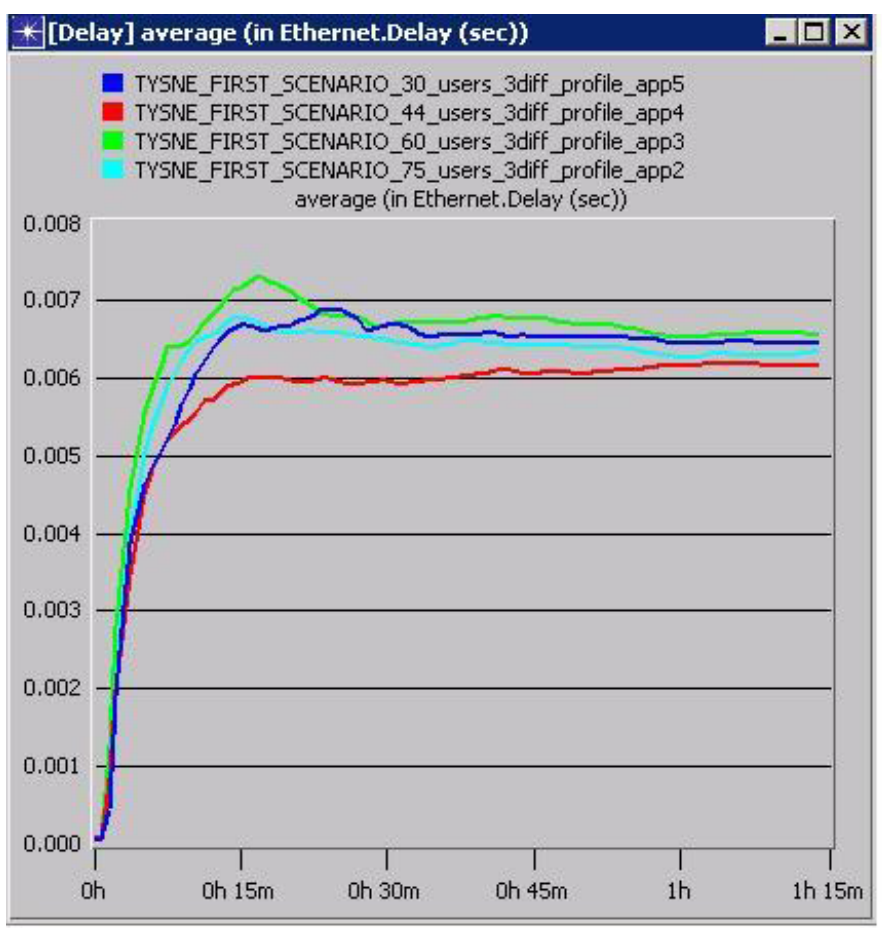

Figure 4: [Delay] average [in Ethernet. Delay (sec)] for four overlaid scenarios.

The simulations scenarios are run allowing the same probability distribution for the start time. The difference in average delay for the scenarios is due to contention among the network 
users on all the intermediate devices and links. Figure 5 gives a picture of the delay variation in seconds as the number of users is augmented.

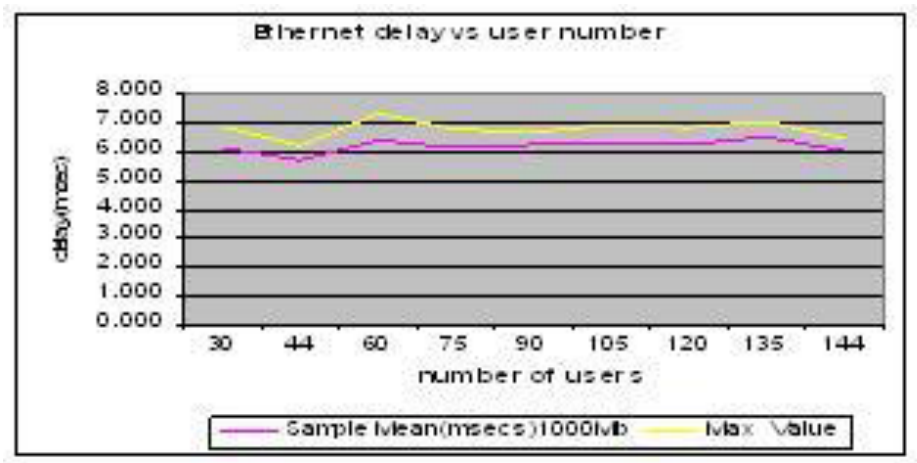

Figure 5: Ethernet average delay vs number of users.

After running simulations for different scenarios and for all the applications considered, increasing number of users, incorporating new number of applications and devices, varying traffic and others we could observe that for this hospital zone ICT network infrastructure there are no problems concerning channel utilization.

Ethernet delay showed to be low with a high throughput. Then, the network prepared to support a greater number of users, more and new applications, as voice, images, video and others.

\section{Conclusions}

Through the case studied, we observe for data transmission that there is no connection with human perception. Even though from human perspective showed discontent about the access and ubiquity. QoS parameters are good.

However, users complain about the straightforward access, new applications and number of computers. In this respect, it is possible to conclude that if the delay is low and throughput is high, there should not be any availability problem, but one of the reasons could be caused of unbalanced resources distribution, some resource policy distribution, or other.

From the various simulations results we conclude that the model is able to detect whether there is connection between human perception of QoS and the technical metrics associated to the ICT network.

From the technology aspects perspective there is a big potential forthcoming for gaining benefit from of ICT support in healthcare service that has to be analyzed also from financial perspective to optimise the available resources. An increase in the number of workstations, increasing the number of new applications, preparing and training people in informatics and technology is a tactic that contributes and leads to increase the usage of technology in the Healthcare sector, mainly in rural communities.

\section{Bibliography}

[1] Elske Ammenwertha, ${ }^{*}$, Stefan Gräberb, Gabriele Herrmannc, Thomas Bürkled, Jochem Königb, Evaluation of health information systems-problems and challenges, INT JOURNAL OF MEDICAL INFORMATICS 71, 125-135, 2010. 
[2] E. Babulak, Quality of service provision assessment in the healthcare information and telecommunications infrastructures, INTERNATIONAL JOURNAL OF MEDICAL INFORMATICS, 75(3):246-252, 2006.

[3] Networking Health: Prescriptions for the Internet, Computer Science and Telecommunications Board, National Academic press, ISBN-10: 0-309-06843 , 2000.

[4] Triantaphyllou, E.. " Multi-Criteria Decision Making Methods: A comparative Study" (Applied Optimization, Volume 44) Nov 2000

[5] Sun, L., and Ousmanou, K., Articulation of Information Requirements for Personalized Knowledge Construction, JOURNAL OF REQUIREMENTS ENGINEERING, 11(4):279293, 2006.

[6] Optimized Network Engineering Tool (OPNET). www.opnet.com, Opnet: User's Manual, http://www.opnet.com/university_program/teaching_with_opnet /textbooks_and_materials/materials/OPNET_Modeler_Manual.pdf, 2004.

[7] OPNET documentation V.11.O.A, OPNET Technologies, Inc., Bethesda, MD, 2004.

[8] Heath A., Carrasco R.( 2001), Access techniques for 3G multimedia wireless packet switched networks: simulation using OPNET, IEE/IEEE/BCS 6th International Symposium on Communication Theory and Applications (ISCTA01), Lancaster University, 15-20 Jul 2001.

[9] Oddershede, A.M, Carrasco, R.A E. Barham. Multicriteria Decision Model for Assessing Health Service Information Technology Network Support using AHP, IBEROAMERICAN JOURNAL OF COMPUTING, Computacion y Sistemas, ISSN 1405-5546, 12 (2): 173-182, 2008

[10] Oddershede, A.M, Carrasco, R.A, Information and Communications Technology Significance in Health Care: User Perception, MEDITERRANEAN JOURNAL OF ELECTRONICS AND COMMUNICATIONS, 2(2): 82-89, 2006. 